\title{
Diálisis peritoneal aguda automatizada en el manejo del síndrome hemolítico urémico
}

\author{
Felipe Cavagnaro S.M. '; Jaime Muñoz P.'; José I. Rodríguez C. '; Ricardo Ronco M. ${ }^{\text {'; }}$ \\ Sandra Vilches J., ${ }^{3}$ Edda Lagomarsino F. ${ }^{1}$
}

\begin{abstract}
Resumen
Objesiro. evaluar las ventajas del sisteme de diálisis peritoneal agudo automatizada en comparación con el procedimiento manual en niños con sindrome hemolíico Jismico. Mérodo: entre 30 pacienies [Edad, $\bar{x}=20,4$ meses] aterididos por sincicme herrolíico urémico en un periodo de 75 meses jerero 1990 o marzo de $997 /$ en un hospital univerzitario de Sanlicgo, Chite, 15 reçuirieron did̆lisis peritoneal y en 1 i se emp é el mélodo altomatizado y en los otros 4 el monu. Resurtados: los pacientes somelidos c pracadimiento manucl registracon 71,3 atenciones diarias de enfermeria en promedio sćlo para el rane c de la diónisis. en comparación con 5,6 atenciones para les lictados ccn el procedimierib au:omálico de ciclado, en el que no se registraron complicac ones Conciusion:es: lo mitad de los pacientes con sindrome hemolitice urémico requirio dálisis pertoneal aguda. La outomalizoción del procedimiento reduce los costes haspitalorios al abreviar a permanenara de los pocientes en uridades de cuidados especiales, mejorar su contacto con sus familares, disminur el número de atenciones de erferr eria, las -ecesidades de enfermeras $y$ el riesgo de infecciones asociados al procedimiento.
\end{abstract}

'Palabras clave: diálısis, pe'roneal. automatizadc, sindrome hemolitico urém co.j

\section{Automated acute peritoneal dialysis in hemolytic uremic syndrome}

Hempytic uremic syndrcme (HUSi is une of the nost commen ccuse of oiguric acule reval icilure in Chile and in South America. Early uti.ization of renal replacement has improved cutcome its. Acute seritoneal dialysis is ten of used for this purpase. Although, it is easv to use. this rrethod is time consuming, mainly regarding patient's nursing care, thus lengtrening the 'respitalization ime in KU FCilites This manuscript examines the use $\sigma^{*}$ the automated peritoneal dialysı (APDI. in po!ients with HUS During 75 months throm janulary 990 to march 1997 ) 30 patients loge $x=20.4$ months, with तils were oarritted to the peciotric words of a university associated rospital. Filteen thildrer. required feritonea dialysis and $11 / 15$ utilizec APD. Trose potents that underwent manual peritoneol ciaysis [MOD' -eeded >1 3 daily nursing assislance, onily for diclytic hand irg, corrpared with 5.6 for those with AP?. There were no comblications associoled with the use of AFD. We corclude that he use SF APD sirongly ameliorales 1re nursing work. moking feasible on earlier discharge of the patın: from line KU and, probcbly, allowing on importan cecrease in hespiralization costs

(Key words: hemolytic uremic syrdrome, pericneal dialysis, ac ste renol failure.)

1. Departamento de Pediatria, Escuela de Medicina, Ponificicia Universidad Católica de Chile.

2. Servicio de Pediatria, Hospital Las Higueras de Tatcahuano

3. Enternera Dialisis, Servicjo de Pediatría, Hospital Clinico Universidad Católica de Chile.
El síndrome hemolítico urémico continúa siendo una de las causas más frecuentes de insuficiencia renal aguda oligúrica en lactantes y preescolares en muchos países sudamericanos ${ }^{\mathrm{l} .} 2$ y en algunas áreas del hemisferio norte ${ }^{3.4}$. Su letalidad, inicialmente alta, ha descendido en la 
última década a menos de $5 \% 4,6$. Uno de los factores que más ha influido en la sobrevida es la sustitución de la función renal con procedimientos de diálisiss, ${ }^{5,}$. El más empleado en niños ha sido la diálisis peritoneal aguda, debido a su fácil instalación y manejo, a sus características discretas y continuas, y a la baja tasa de complicaciones, en comparación con la hemodiá. lisis. Uno de sus inconvenientes es la alta demanda de atenciones de enfermería que origina para su ejecución continua, en ciclos (período comprendido entre dos drenajes) cortos, usualmente horarios, obligando a realizarla, por lo general, en unidades de cuidados intensivos.

Las máquinas programables de diálisis peritoneal (cicladoras) son capaces de controlar la secuencia temporal de los pasos de la diálisis con gran independencia del paciente $y$ sin necesidad de atención permanente de enfermería. Ellas se han empleado casi exclusivamente en pacientes con afecciones renales crónicas. logrando mejorar su calidad de vida y disminuir la incidencia de complicaciones ${ }^{8}$.

El propósito del análisis que se oferece en seguida fue evaluar los beneficios clínicos y económicos de la diálisis peritoneal automatizada (mediante el empleo de un sistema de ciclado) en pacientes pediátricos con síndrone hemolítico urémico. Esta enfermedad es un buen modelo, porque en ella no suelen ocurrir daños importantes de otros sistemas que obliguen mantener al paciente en unidades do cuidados intensivos por razones ajenas a la falla renal.

\section{Pacientes y Métodos}

Se revisaron. retrospectivamente las fichas clínicas de los pacicntes hospitalizados con el diagnóstico de síndrome hemolitico urémico on los servicios de pediatria $y$ de euidados intersivos pediatricos del Hospital Clinico de la Pontificia Universidad Católica de Chile entre enero de 1990 y marzo de 1997 (75 meses). En los pacientes que reuuirieron diálisis peritoneal sc obtuvo información sobre la causa por la que sé indicó el procediniento, la mordalidad de este (matbual o automatica). duración y complicaciones. En rodos los casos el catérer pcritoneal era de típo Tenckholf, en espiral, con un manguito, y fue instalado en pabellón quirúrgico por un cirujano pediátrico usando un protocolo que incluye omentectomja parcial. Tan pronto Ios pacientes reingresaron a la unidad de cuidados intensiros se injeio la diálisis manualmente. Desde abril de 1994 se cmpleó una cicladora (Pac-Xıra, Baxier Healthcare Co. IIlinojs, USA), a Io que Ios cnfermos fueron conecrados dentro de las 24 horas siguientes al comienzo de la diálisis.
Si las condiciones clínicas del paciente lo permitían, eran retirados de la unidod de cuidados intensivos y trasladados a la sala pediátrica para continuar la peritoneodiálisis. Las inditaciones de tiempo y la composición de los líquidos de diálisis fueron similares en los pacientes dializados con método rnanual y automático, reflejando sus necesidades individuales. El catéter peritoneal era retirado dentro de las 48 boras de terminada la diálisis, después de comprobar que la diuresis fuese mayor a $0.7 \mathrm{ml} \cdot \mathrm{kg} \cdot \mathrm{h}$ (con o sin diuréticos) y estabilidad o mejoria de los criterios bioquimicos de función tenal. Como dato complementario, y para evaluar los requerimientos de atención de enfermeria sobre ambas técrica de DPA (manual y automatizada). en dos pacientes de tada grupo se contabilizó, por tres días consecutivos, el número de alenciones diarias de enfermería consignadas en hoja de enfermería y dirigidos exclusivamente al manejo de lá djálisis.

\section{Resultados}

Se registraron 30 pacientes con síndrome hemolítico urémico durante el período del estudio (promedio de 4,8 pacientes admitidos con la afeccion por cada año). La edad promedio fue de 20.4 meses (márgenes 4 a 59 meses), $68 \%$ de sexo femenino. En 93,3\% (28/30) hubo diarrea como pródromo, generalmente de características disentéricas. Sólo en 6 pacientes se aisló Escherichia coli enteropatógena del cultivo de deposiciones, pero las cepas no fueron tipificadas. Quince pacientes (50\%) requirieron diálisis peritoneal, principalmente por oljguria ( $>24$ horas), anuria o alteraciones metabólicas severas (azotemia, hiperkalemia). En 11 (73\%) se aplicó el procedimiento automatizado, en su totalidad en los últimos 36 meses del período. Las características demográficas y antropométricas de los niños que requirieron diálisis no se diferenciaron estadísticamente del total de afectados por la enfermedad.

La duración de la hospitalización en los pacientes sometidos a diálisis automatizada fue (promedio) 16,I días (márgenes 8 a 30 días), y la permanencia (promedio) en la unidad de cuidados intensivos fue 4,6 días (márgenes 2 a 12 días). Siete $(64 \%)$ de estos pacientes estuvieron 4 o menos días en la unidad de cuidados intensivos (promedio 2,7 días). De los otros 4 pacientes, 2 sufrieron daño intestinal severs y requirieron hemofiltración continua (uno antes y otro después de la diálisis peritoneal ${ }^{9} ;$ los otros 2 pacientes permanecieron en cuidados intensivos por decisión del equipo tratante para mejor vigilancia hemodinámica. En todos los casos en que 
se us6, los pacientes fueron conectados a la cicladora dentro de las 24 horas de iniciada la diálisis manual y trasladados a sala pediátrica posteriormente. El promedio del tiempo en diálisis automatizađa fue 10,3 días (márgenes 5 a 18 dias). Ningún paciente retornó a diálisis manual o a cuidados intensivos luego de este periodo.

En los 4 pacientes sometidos exclusivamente a diálisis peritoneal manual, el tiempo promedio de hospitalización fue de 18,5 días (márgenes 12 a 24 días) y la diálisis duró un período promedio de 14,2 días (márgenes 4 a 22 días). El número de atenciones diarias de enfermería relacionados exclusivamente con la diálisis fue 71,3, comparado con sólo 5,6 en el procedimiento automático. En la modalidad manual se emplearon 7,4 atenciones (promedio) diarias para apertura del sistema por cambio de bolsas de solución dialítica, en la automatizada sólo 1,2 .

Dos pacientes sufrieron por dificultades con el flujo de salida del líquido peritoneal, que en un caso obligó a suspenderla. En otros cuatro se registraron filtraciones de líquido de diálisis alrededor del sitio đe salida del catéter, siendo necesario cambiar el catéter en uno. Un paciente presentó un episodio de peritonitis el mismo día en que se suspendio la diálisis (día 15), oplándose por dejar el catéter otros 10 días para tratamiento intraperitoneal con antibióticos. En la evaluación clínjca de seis meses después del alta, en nueve pacientes que han completado este período de seguimiento, la creatinina plasmática era normal en todos y sólo un paciente tenía proteinuria leve.

\section{Comentario}

El promedio anual de casos de síndrome hemolítico de este estudio casi duplica al de años anteriores en el mismo servicio ${ }^{10}$, to cual se puede deber a mejor diagnóstico de casos leves 0 incompletos ${ }^{2}$ y a mayor referencia de pacientes desde otros centros de salud. Si bien probablemente debido al sesgo mencionado el requerimiento por diálisis resulta más alto que en otras experiencias locales ${ }^{10}$, pero semejante a 10 descrito en otros paises ${ }^{11,12}$.

Nuestro equipo, desde hace algunos años, hace instalar el catéter peritoneal en pabellón, por cirujano infantil entrenado, quien fija el manguito al peritoneo y aboca el sitio de salida alejado por contraabertura. Este procedimiento estándar ha permitido disminuir la incidencia de daño accidental a vísceras intraabdominales, peritonitis asociadas al catéter y filtraciones de líquido alrededor del sitio de salida, y es el de elección cuando no se conoce la duración que tendrá de la diálisis aguda ${ }^{13.14}$. La omentectomía parcial parece reducir el riesgo de pérdida de catéteres por obstrucción ${ }^{15}$.

El tiempo de diálisis necesario para alcanzar los criterios de término de esta (10,3 días) fue semejante al de otras series ${ }^{11,16}$ y mayor que el descrito como conveniente ( 3 días) para recambiar catéteres agudos, en consideración al aumento exponencial del riesgo de peritonitis ${ }^{13}, 17$. En nuestro equipo el solo término de la anuria no constituye razón suficiente para suspender la diálisis, ante el riesgo de tener que reinstalarla posteriormente por necesidades de espacio intrayascular para infusión de drogas o transfusiones, alteraciones bioquímicas severas propias del síndrome urémico, y porque ello obliga a importantes restricciones de líquidos, calorías y proteínas en niños que están muchas veces hipercatabólicos y/o mal nutridos.

Si se asume que todo paciente sometido a diálisis peritoneal aguda debiera permanecer, por las razones antes recién señaladas, en una unidad de cuidados intensivos o intermedios, como suele ocurrir con el procedimiento, la diálisis automatizada economiza, promedio, 5,7 días de hospjtalización en unidades de mayor complejidad, cifra que podría mejorar aun más si consideramos que, en estricto sentido, solo dos pacientes tenían que permanecer en ellas por complicaciones extrarrenales severas. Este hecho puede constituir un significativo ahorro en costos de hospitalización de este tipo de pacientes, al tiempo que se permite a los padres permanecer más tiempo junto a sus hijos hospitalizados. Por otro lado, las atenciones de enfermería relacionadas con el procedimiento automatizado constituyen $56108 \%$ de las originadas por el método manual. Conclusiones similares han sido recientemente planteadas por otros autores ${ }^{18}$. De acuerdo con el número de veces que el sistema debió ser abierto para cambio de bolsas de diálisis, el riesgo de peritonitis en los pacientes en diálisis manual sería eventualmente seis veces mayor que en el modo automático. 
Este riesgo, poco estudiado en la práctica ${ }^{19}$, pareciera sugerido en publicaciones que comparan estos métodos en pacientes renales crónicos ${ }^{20}$. El único paciente que desarrolló peritonitis en esta serie lo hizo cuando ya no se requerían intercambios de líquido peritoneal.

\section{Referencias}

1. Cordovez A. Prado V. Maggi L, et al: Enterohemonhagic Escherichia coli associated with hemolyticuremic syndrome in chilean children. J Clin Microbiol 1992; 30: 2153-2157.

2. López E, Contrini $M$. Devoto $S$ et al Incomplete hemolytic-uremic syndrome in argentinean children with bloody diarthea. J Pediatr 1995: 127: 364-367.

3 Loirat Ch. Baudouin V. Sonsino E, Mariani-Kurdjian $P$, Elion $J$ : Hemolytic uremic syndrome in the child. Advances in Nephrology $1993 ; 22 ; 141-167$.

4 Grimm PC. Ogburn MR: Hemolytic uremic syndrome: The most common cause of acute renal failure in childhood. Pediatr Aan 1994, 23: 505-51 l.

5. Robson WL, Leung AK, Kuplon BS: Hemolytic-nremic syndrome. Curr Probl Pediatr 1993: 23: 16-33

6. Pickering $L$, Obrig $T$. Stapleton $B$ : Hemolytic-uremic syndrome and enterohemorrhagic Escherichia coli. Pediatr Infect Dis J 1994; 13: 459-476.

7. Kaplan B, Levin M, De Chadarevian JP: The hemolytic uremic syndrome. In: Edelman Ch (ed.): Pediatric Kidney Disease. $2^{\text {a }}$ edition, Little, Brown and Company, Boston, 1992: 1383-J405.

8. Castro M, Hevia C, Sánchez $S$ et al: Medium tem experience with "Home Choice" for automated peritoneal dialysis (APD) at one center. Perit Dial Int 1997; 17 (suppl I):\$93

9. Cavagnaro $F$, Ronco $R$, Verdaguer $M$, Dfaz JP, Levin $L$, Cerda $M$ : Continuous hemofiltration in children with abdominal complications of hemolytic-uremic syndrome. Nephron 1996; 74: 433-434.
10. Cavagnaro $F$, Lagomarsine $E$, Gonzalez $C$, GarctaHaidobro I. Cerda M: Hemolytic uremic syndrome: Comparison of clinical and laboratory parameters between two periods separated ten years. Pediatr Nephrol 1995; 9:112.

11. Brandf J, Fouser L, Watkins $S$, et al: Escherichia coli 0157:H7-associated hemolytic-uremic syndrome after ingestion of contamined hamburgers. J Pediatr 1994 124: 519-526.

12. Hall $S$, Glickman $M$ : The British Paediatric Survejllance Unit. Arch Dis Child 1988; 63:344-346.

13. Ash S. Daugirdas $J$ : Peritoneal acces devices. In: Handbook of Dialysis, Daugirdas J y Todd Y (eds), $2^{\text {a. }}$ edition, Listle, Brown and Company, Boston, 1994 274-300.

14. Wong S, Geary D: Comparison of temporary and permanent calheters for acute peritoneal dialysis. Arch Dis Child 1988; 63: 827-831.

15. Pumford N, Cassey J, Uttley W: Omentectomy with peritoneal catheter placement in acute renal failure Nepbron 1994; 68: 327-328.

16. Spizirri $F$, Rahman $R$ Bibiloni $N$, Ruscasso J, Antoreo $O$ : Childhood hemolytic uremic syodrome in Argentina: long-term follow up and prognostic features. Pedintr Nephrol 1997; 17: 156-160.

17. Day $R$, White $R$ : Peritoneal dialysis in children: review of 8 year's experience. Arch Dis Child 1977; 52:5661 .

18. Vande Walle J. Raen A. Castillo D. Lutz-Dettinger N: New perspectives for peritoneal dialysis in acute renal failure, related to new catheter techniques and introduction of cycler. Perif Dial Int 1997; 17 (suppl 1): \$90.

19. Kohli $H$, Arora P. Kher V. Gupta A, Sharma $R$. Bhoumik $S$ : Daily peritoneal dialysis using a surgically placed Tenckhoff catheter for acute renal failure in children. Ren Fail 1995; $17 ; 51-56$.

20. De Fijter $C$. Verbrugh $H_{4}$ Oe $L$, el al: Peritoneal defense in continuous ambulatory versus continuous cyclic peritoneal dialysis. Kidney lnt $1992 ; 42: 947$. 950.

21. Valenzuela A, Lagomarsino E, Cauagnaro $F$, Solar E: Insuficiencia renal crónica en la infancia. Encuesta nacional, Rev Chil Pediatr 1996: 67: 116-120. 